\title{
New insights into the clinical and molecular spectrum of the novel CYFIP2-related neurodevelopmental disorder and impairment of the WRC-mediated actin dynamics
}

\author{
A full list of authors and affiliations appears at the end of the paper.
}

\begin{abstract}
Purpose: A few de novo missense variants in the cytoplasmic FMRP-interacting protein 2 (CYFIP2) gene have recently been described as a novel cause of severe intellectual disability, seizures, and hypotonia in 18 individuals, with p.Arg87 substitutions in the majority.
\end{abstract}

Methods: We assembled data from 19 newly identified and all 18 previously published individuals with CYFIP2 variants. By structural modeling and investigation of WAVE-regulatory complex (WRC)-mediated actin polymerization in six patient fibroblast lines we assessed the impact of CYFIP2 variants on the WRC.

Results: Sixteen of 19 individuals harbor two previously described and 11 novel (likely) disease-associated missense variants. We report p.Asp724 as second mutational hotspot (4/19 cases). Genotype-phenotype correlation confirms a consistently severe phenotype in p.Arg87 patients but a more variable phenotype in p. Asp724 and other substitutions. Three individuals with milder phenotypes carry putative loss-of-function variants, which remain of unclear pathogenicity. Structural modeling predicted missense variants to disturb interactions within the WRC or impair CYFIP2 stability. Consistent with its role in WRC-mediated actin polymerization we substantiate aberrant regulation of the actin cytoskeleton in patient fibroblasts.

Conclusion: Our study expands the clinical and molecular spectrum of CYFIP2-related neurodevelopmental disorder and provides evidence for aberrant WRC-mediated actin dynamics as contributing cellular pathomechanism.

Genetics in Medicine (2021) 23:543-554; https://doi.org/10.1038/s41436020-01011-x

Keywords: intellectual disability; epilepsy; CYFIP2; WAVEregulatory complex (WRC); WASF

\section{INTRODUCTION}

CYFIP2 encodes the cytoplasmic fragile $\mathrm{X}$ mental retardation protein (FMRP) interacting protein 2 . It is thought to play an important role in neurodevelopment by linking FMRPdependent local transcription with signaling-dependent remodeling of the cytoskeleton as part of the WAVE-regulatory complex (WRC). ${ }^{1}$ The WRC is a key regulator of actin dynamics and consists of WASF (also known as WAVE), CYFIP1/2, NCKAP1, ABI, and BRICK1 (or their homologs). In the basal state the heteropentameric WRC is intrinsically inhibited by CYFIP due to the sequestration of the activitybearing VCA domain of WASF. Binding of the small GTPase RAC1 to CYFIP induces conformational changes of the WRC, which release the VCA domain to trigger Arp2/3-induced actin polymerization at distinct membrane sites. ${ }^{2}$ Accordingly, the WRC signaling pathway plays a crucial role in important neurodevelopmental processes such as axon guidance and regulation of synapse morphology. ${ }^{1,3}$

Recently, de novo missense variants affecting a small number of amino acids and one late-truncating variant in CYFIP2 have been associated with intellectual disability (ID), seizures, and muscular hypotonia (MIM 618008). ${ }^{4,5}$ Initially, in 2018
Nakashima et al. described three de novo CYFIP2 variants in four unrelated individuals with early-onset epileptic encephalopathy, all affecting the arginine at position 87 , and suggested a gain-of-function effect on the WASF signaling pathway. ${ }^{4}$ Subsequently, we reported that not only p.Arg87 variants, but also a variety of spatially clustering de novo variants are the cause of a new neurodevelopmental disorder characterized by intellectual disability and seizures in 12 patients. ${ }^{5}$ We predicted all of the detected variants to decrease the stability of the WRC, thereby reducing the inhibitory effect of CYFIP2 on WASF activity and eventually resulting in a gain of function of the WASF pathway. ${ }^{5}$ With two additional reported individuals harboring recurrent p.Arg87 variants, a total of only 18 individuals with CYFIP2 variants have been described to date. ${ }^{6,7}$

In this study, we considerably increase the number of individuals harboring a variety of CYFIP2 variants by reporting 19 additional cases. Genotype-phenotype correlation confirms a profound phenotype for substitutions at the mutational hotspot p.Arg87 and demonstrates a more variable phenotype associated with a novel recurrent variant and with several unique missense alterations, while there is currently no evidence that loss-of-function variants lead to a similarly

Correspondence: Anita Rauch (anita.rauch@medgen.uzh.ch)

These authors contributed equally: Markus Zweier, Anita Rauch 
severe phenotype. Our studies in primary fibroblast cultures of six affected individuals demonstrate that the WRCmediated actin dynamics are disturbed by the CYFIP2 missense variants.

\section{Patients}

\section{MATERIALS AND METHODS}

The herein described cohort of 19 novel individuals (I1-I19) was recruited through personal communication following our initial report ${ }^{5}$ and the GeneMatcher Database ${ }^{8}$ from Germany, France, the United Kingdom, the United States, Canada, Brazil, and New Zealand. The CYFIP2 variant was identified by trio-exome sequencing (individuals $1,2,3,4,6$, $7,8,9,10,12,13$, and 18) or single-exome sequencing with subsequent parental testing by Sanger sequencing (individuals $5,11,14,17$, and 19). Individual 16 and his mother as well as individual 15 underwent sequencing of the autism/intellectual disability (ID) Xpanded panel from GeneDx, with subsequent Sanger sequencing of the father of individual 15. All reported exonic variants are described according to the CYFIP2 reference transcript NM_001291722.1 encoding for NP_001278651.1, in line with the current HGVS nomenclature (v19.01, varnomen.hgvs.org). Intronic variants are named based on the genomic reference sequence NC_000005.10. All reported variants have been submitted to the Leiden Open Variation Database (LOVD) accessible at www.LOVD.nl/ CYFIP2 (individual IDs 00301307-00301325).

\section{Ethics statement}

This study was performed as part of a research study approved by the ethics commission of the Canton of Zurich (ID PB_2016-02520 [SIV 11/09]). Genetic testing in collaborating centers was performed either in the setting of routine diagnostic testing without the requirement for institutional ethics approval or within research settings approved by the ethical review board of the respective institutions (Ethics Committee of the Medical Faculty of the University of Bonn, approval numbers 131/08 and 024/12; DDD ethics approval from NHS REC Cambridge South, approval number 10/ H0305/83; Ethics Committee of the Dijon University Hospital, approval numbers ID RCB: 2016-A01347-44 and CPP EST I: 2016/38). Written informed consent for genetic testing, for publication of mutational and clinical data, and for publication of patient photos was obtained from the individual's parents or legal guardian.

\section{RNA analysis}

RNA was extracted from whole blood of individual 17 and six controls using the PAXgene Blood RNA Kit (PreAnalytiX, Hombrechtikon, Switzerland) and transcribed reversely using SuperScript III First-Strand Synthesis System (ThermoFisher Scientific, Waltham, MA, USA). PCR on complementary DNA (cDNA) was performed using specific primers located in exons 18 and 22 of CYFIP2 and the products were analyzed by agarose gel electrophoresis. Distinct bands were extracted using the QIAquick Gel
Extraction Kit (Qiagen, Venlo, the Netherlands) and analyzed by Sanger sequencing.

\section{Structural variant modeling}

The effect of the variants was modeled in silico on the basis of the crystal structure of the WAVE-regulatory complex as previously described. ${ }^{5}$ RasMol $^{9}$ was used for structure analysis and visualization. The effect of the variants on CYFIP2 stability and binding affinity to the other WRC proteins was assessed using BindProfX ${ }^{10}$ and FoldX. ${ }^{11}$

\section{Fibroblast cultures}

Primary fibroblast cultures were established from skin biopsies of individual 8 (p.(Asp724Tyr)), individual 9 (p.(Asp724Gly)), and individual 11 (p.(Glu468Asp)) of the current study as well as of P1 (p.(Arg87Cys)), P6 (p.(Ile664Met)), and P12 (p.(Tyr108His)) previously reported by Zweier et al., ${ }^{5}$ and of three unaffected individuals as controls. Fibroblasts were cultured in DMEM supplemented with $10 \%$ fetal bovine serum and $1 \%$ penicillin-streptomycin (all Gibco, Life Technologies, Carlsbad, CA) and tested negative for infection with Mycoplasma.

\section{Migration assays}

Wound healing assays were performed three times in duplicates using black glass bottom 24-well plates with culture inserts (ibidi, Gräfelfing, Germany). Ten thousand fibroblasts were seeded in both wells of the insert in full medium and cells were grown to a confluent monolayer for 24 hours. A consistently sized gap was created by removing the inserts; cells were washed and incubated in medium supplemented with $10 \mathrm{pg} / \mathrm{ml}$ of human platelet derived growth factor BB (PDGF) (P3201, Sigma-Aldrich, St. Louis, Missouri). Migration was assessed by multiframe live cell imaging at $37^{\circ} \mathrm{C}$ and $5 \% \mathrm{CO}_{2}$ every 30 minutes for 24 hours with a $5 \times$ objective on a Zeiss AxioObserver Z1 widefield microscope. Images were batch processed by enhancing contrast before using a pipeline in Ilastik ${ }^{12}$ for segmentation into cell-free and cell-covered area, which was then quantified using CellProfiler. ${ }^{13}$ The cell-free area was plotted in GraphPad Prism 8.3 (GraphPad Software, La Jolla, CA) and values in the linear phase (from 0 to 15 hours) were fitted with a linear regression, which was used to calculate the time to half gap and migration speed. Statistical significance of differences in relation to three controls was calculated using one-way analysis of variance (ANOVA) and a significance level of $p<0.05$.

\section{Phalloidin staining}

Fibroblasts were seeded on Poly-D-Lysine coated coverslips in 24-well plates and allowed to attach for about 1.5 hours before changing to a serum-free medium for 24 hours. Cells were stimulated with $100 \mathrm{ng} / \mathrm{ml}$ PDGF in full medium at $37^{\circ} \mathrm{C}$ for 5 minutes, then were washed with warm phosphate-buffered saline (PBS) and fixed with 3.7\% methanol-free formaldehyde for 10 minutes at room temperature. For the staining, cells 
were permeabilized with $0.1 \%$ Triton X-100 for 15 minutes at room temperature, incubated with phalloidin conjugated to Alexa Fluor 568 for 60 minutes at room temperature, counterstained with DAPI, and mounted with ProLong Gold Antifade Mountant (ThermoFisher Scientific). Images for quantification of dorsal ruffles were taken on a Zeiss AxioObserver $Z 1$ with a $25 \times$ oil objective. Experiments were repeated three times and at least 200 cells were counted for each coverslip. Data were analyzed using Prism 8.3 (GraphPad Software, San Diego, CA, USA). Statistical significance of differences in relation to three controls was calculated using one-way ANOVA and a significance level of $p<0.05$.

\section{RESULTS}

We identified 16 individuals with likely disease-associated missense variants in CYFIP2 and three individuals with putative loss-of-function (LoF) variants of unknown significance (Table 1).

\section{A novel recurrent variant and several unique missense variants are associated with variable phenotypes}

The 16 individuals with likely disease-associated missense variants harbored 11 novel and two recurrent substitutions in CYFIP2 (Fig. 1a). Three of these carry the previously reported hotspot variant p.(Arg87Cys), ${ }^{4-6}$ and two harbor novel substitutions of the arginine at this position (p.(Arg87His) and p.(Arg87Ser)). Moreover, we establish the aspartate at protein position p.724 as a new recurrently affected residue mutated in four individuals (p.(Asp724His), p.(Asp724Gly), and p.(Asp724Tyr)).

An overview of inheritance mode, conservation, computational predictions, presence in gnomAD, and available experimental evidence is given in Table 1 for all CYFIP2 missense variants. All variants reported in the present study are absent from gnomAD v2.1.1 and affect highly conserved amino acids, except p.(Thr490Met) (individual 12), which was detected once in gnomAD (minor allele frequency of 0.00003185 ) and affects a moderately conserved amino acid. All variants were predicted to be deleterious by SIFT, PolyPhen-2, and MutationTaster, apart from p.(Arg87Ser), p.(Met311Thr), and p.(Met456Val) that show benign predictions in PolyPhen-2. De novo inheritance was confirmed for all 12 individuals where both parental samples were available for testing, while in four individuals only one parental sample could be obtained. According to the guidelines of the American College of Medical Genetics and Genomics (ACMG), 10 variants in 13 individuals were classified as pathogenic or likely pathogenic, while three missense variants (p.(Met456Val) in individual 14, p.(Phe888Ser) in individual 15 , and p.(His1206Tyr) in individual 16) had to be formally classified as variants of unknown significance due to incomplete segregation testing. Nevertheless, based on their absence in control populations, multiple lines of computational evidence supporting deleterious effects, their location in the tertiary structure, and the result of our mutational modeling indicating similar effects as predicted for clearly pathogenic variants, we assume also these three missense variants are likely causative for the phenotype. Thus we considered all 13 missense variants to be likely diseaseassociated variants.

In all individuals of this cohort harboring missense variants developmental delay (DD) was noted (at a median age of 6.5 months; range 6 weeks-2 years): 14/16 with global DD and 2/ 16 with normal motor but delayed language development (Table 2, Supplementary Table S1). Intellectual disability was classified as severe or profound in 9 of 14 evaluated individuals, as moderate in $4 / 14$, and as mild in $1 / 14$ cases (according to Diagnostic and Statistical Manual of Mental Disorders, 5th Edition [DSM V] criteria). Individuals 1 and 4 were too young for evaluation of ID with an age of 1 year 4 months or 5 months, respectively, at last investigation. About half of the individuals in this cohort were reported to have epilepsy (8/16, and suspected in individual 5) with onset at a median age of 5 months (range neonatal-14 years). Generalized tonic-clonic seizures (6/8) and epileptic spasms (4/8) were the most prevalent seizure types. Electroencephalogram (EEG) findings were variable, with hypsarrhythmia reported in $4 / 8$ individuals. Two individuals were seizure-free on antiepileptic polytherapy, while six remained refractory to treatment. Ten of 16 individuals showed generalized or truncal muscular hypotonia, combined with limb hypertonicity in two cases. The majority of individuals presented with a head circumference $(\mathrm{HC})$ in the lower range $(11 / 16$ individuals with $\mathrm{HC}<-1.0 \mathrm{SD}$ score). Microcephaly with a HC below 2 standard deviations was diagnosed in 5/16 individuals of the current cohort. Dysphagia was reported in seven individuals; three of them were dependent on gastric tube feeding. Variable visual problems were present in six individuals, and sleep difficulties were reported in seven individuals. Morphological features revealed a shared but not clearly recognizable facial gestalt including high, narrow forehead; apparent hypertelorism; depressed nasal bridge; bulbous nasal tip; full cheeks; everted lip vermilion; and long tapered fingers in several individuals (Fig. 1b). All individuals are alive at their age at last evaluation ( 5 months to 22 years). A collation of the clinical features from all the cases (Supplementary Table S1) and case reports for each case can be found in the Supplement.

\section{No evidence that loss-of-function variants lead to a similarly severe phenotype}

Three individuals harbored heterozygous CYFIP2 variants for which a (partial) loss of one allele product is expected (Fig. 1a and Table 1). Individual 18 harbored the de novo variant p.(Lys501*) while individual 19 carried the variant p.(Ser258Glnfs*2) with unknown segregation. In individual 17 the splice variant c.2058-1G>C was detected and shown to be absent in the mother. The variant resulted in an aberrant in-frame transcript (lacking 58 codons) and an aberrant out-of-frame transcript (p.(Glu686_Ser744delinsAsp) and p.(Tyr687*), Supplementary Fig. S1). Individual 19 had mild 


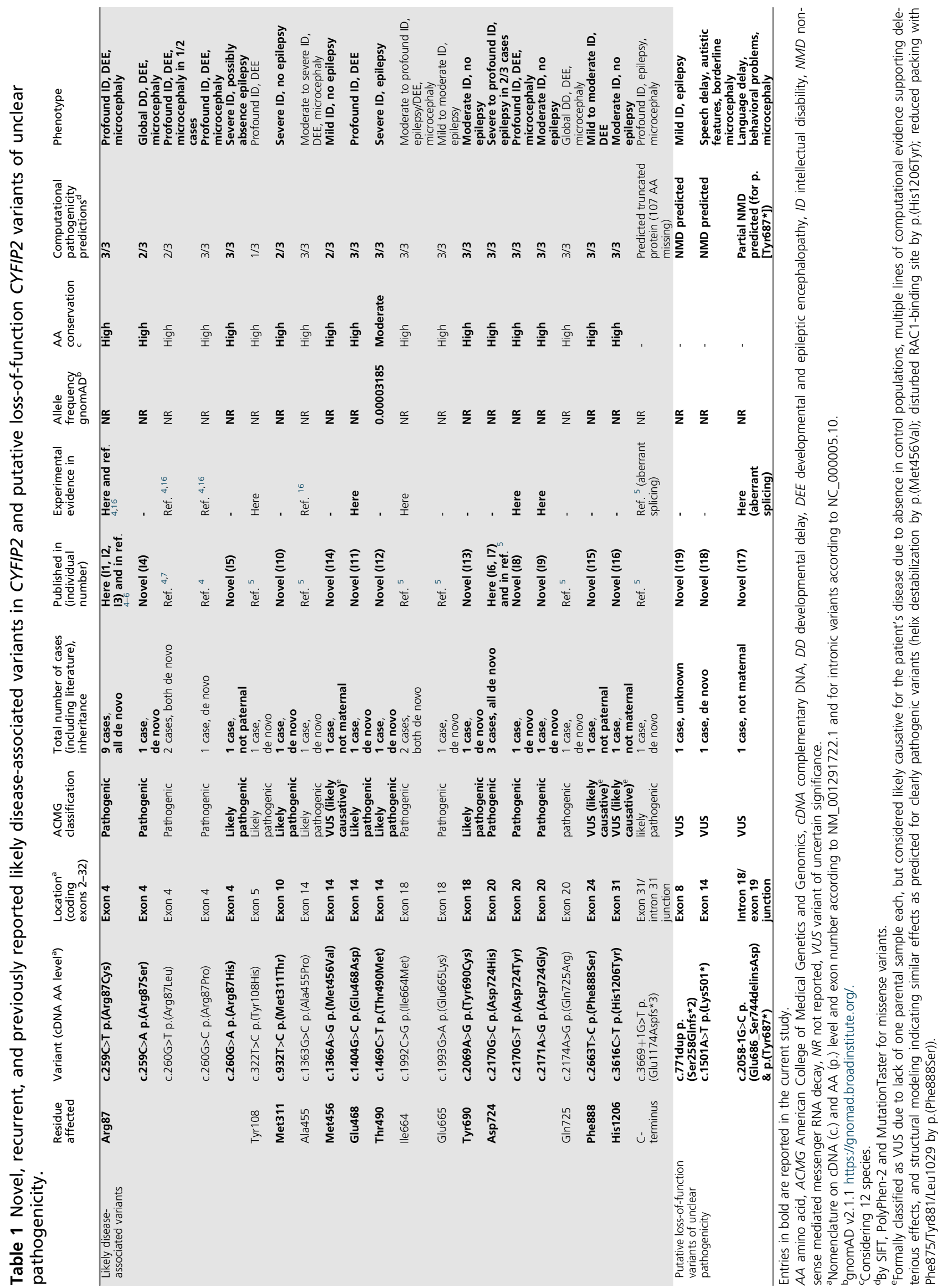



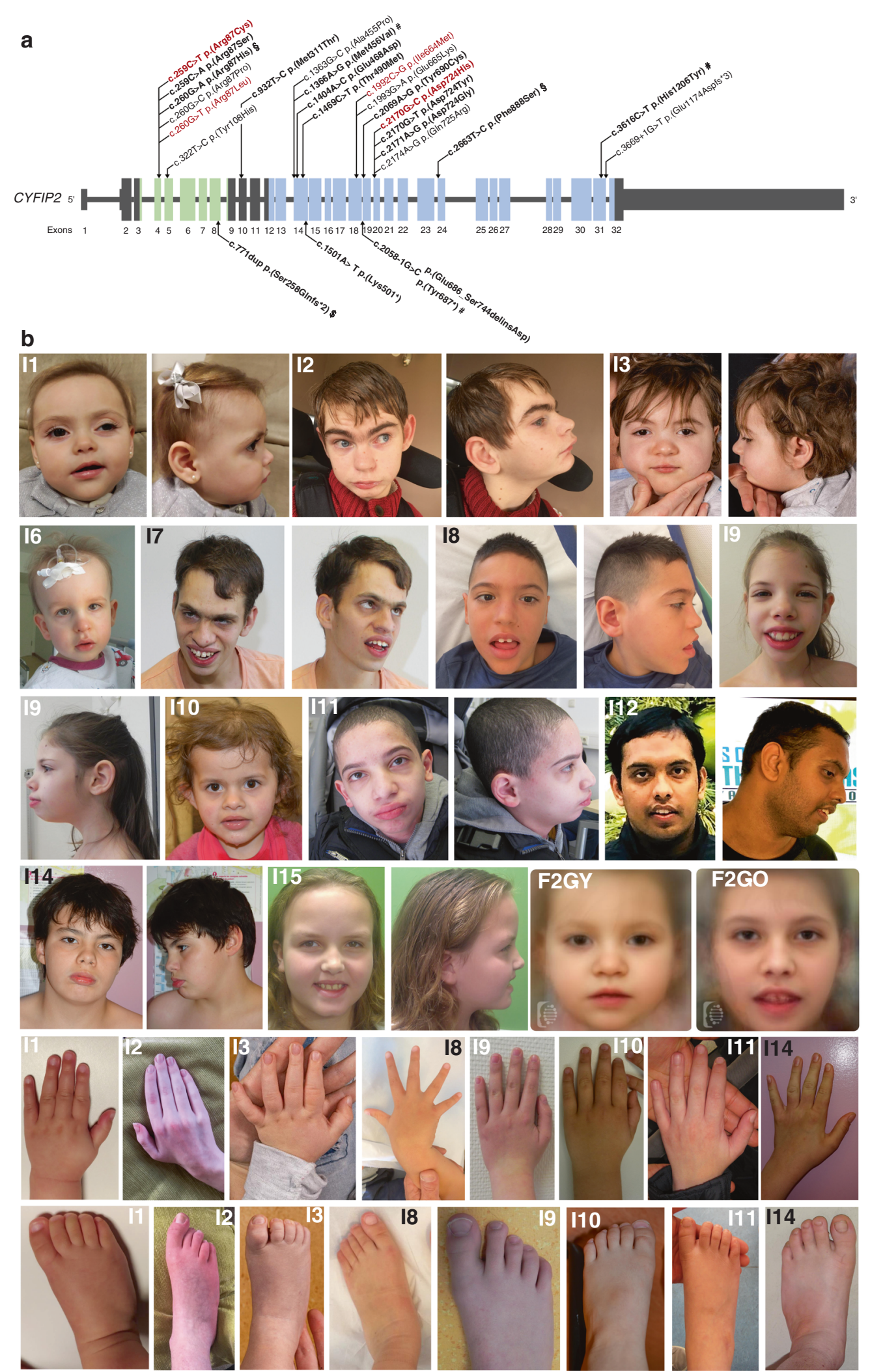

ID and epilepsy while individuals 18 and 17 were both affected by language delay, behavioral problems, and microcephaly. Of note, individuals 18 and 17 had a family history of language delay not segregating with the variant and the parents of individual 19 are consanguineous. A detailed clinical description is provided in the case reports in the Supplement. Note that the clinical data of these individuals are not included in Table 2. 
Fig. 1 CYFIP2 variants and morphological features of affected individuals. (a) Schematic drawing of the CYFIP2 gene in chromosomal region $5 q 33.3$ with known and novel variants identified. (Likely) pathogenic variants are shown above and putative loss-of-function variants are depicted below the gene scheme. Red labeling represents recurrent variants, and bold letters indicate variants detected in the present study. All variants were shown to have occurred de novo except those marked with \# (not maternal), § (not paternal), or \$ (segregation unknown). Variant nomenclature and gene structure according to NM_001291722.1 with exons numbered from 1 to 32 consecutively and NC_000005.10 for intronic sequences. Regions coding for the conserved protein domains DUF1394 and FragX_IP are depicted in light green and light blue, respectively (according to the National Center for Biotechnology Information [NCBI] conserved domain database). (b) Morphological features of individuals with likely disease-associated variants in CYFIP2. Note a shared but not clearly recognizable facial gestalt including high, narrow forehead; apparent hypertelorism; depressed nasal bridge; bulbous nasal tip; full cheeks; everted lip vermilion; and long tapered fingers in several individuals. Composite facial appearance in younger and older individuals is shown in F2GY and F2GO, respectively (created by Face2Gene [FDNA Inc., Boston, MA, USA] with ten photos of nine individuals from this study and Zweier et al. ${ }^{5}$ each).

Table 2 Main clinical features in all CYFIP2 patients with recurrent and unique likely disease-associated variants (published previously and in the current study).

\begin{tabular}{|c|c|c|c|c|c|}
\hline Feature & $\begin{array}{l}\text { Arg87 substitutions } \\
n=15^{\mathrm{a}}\end{array}$ & $\begin{array}{l}\text { Ile664 substitutions } \\
n=2^{\mathrm{a}}\end{array}$ & $\begin{array}{l}\text { Asp724 substitutions } \\
n=5^{a}\end{array}$ & $\begin{array}{l}\text { Non-recurrent sites } \\
n=12^{a}\end{array}$ & $\begin{array}{l}\text { All CYFIP2 patients } \\
n=34^{\mathrm{a}}\end{array}$ \\
\hline ID/DD & $15 / 15$ & $2 / 2$ & $5 / 5$ & $12 / 12$ & $34 / 34(100 \%)$ \\
\hline Severe/profound & 12 & 1 & 4 & 6 & $23(68 \%)$ \\
\hline Moderate & 0 & 1 & 1 & 4 & $6(17 \%)$ \\
\hline Mild & 0 & 0 & 0 & 1 & $1(3 \%)$ \\
\hline $\begin{array}{l}\text { Too young to } \\
\text { classify }\end{array}$ & 3 & 0 & 0 & 1 & $4(12 \%)$ \\
\hline $\begin{array}{l}\text { Behavioral } \\
\text { problems }\end{array}$ & $3 / 8$ & $1 / 2$ & $2 / 5$ & $9 / 12$ & 15/27 (56\%) \\
\hline Epilepsy & $15 / 15$ & $1 / 2$ & $2 / 5$ & $8 / 12$ & 26/34 (76\%) \\
\hline Microcephaly ${ }^{b}$ & $10 / 14$ & $2 / 2$ & $1 / 5$ & $3 / 12$ & $16 / 33(48 \%)$ \\
\hline $\begin{array}{l}\text { Muscle tone } \\
\text { anomalies }\end{array}$ & $12 / 14$ & $2 / 2$ & $5 / 5$ & $7 / 12$ & $26 / 33(79 \%)$ \\
\hline cMRI anomalies & $9 / 14$ & $0 / 1$ & $1 / 5$ & $4 / 12$ & $14 / 32(44 \%)$ \\
\hline Visual problems & $7 / 9$ & $1 / 2$ & $3 / 5$ & $4 / 12$ & $15 / 28(54 \%)$ \\
\hline Feeding difficulties & 9/11 & $0 / 2$ & $3 / 5$ & $3 / 12$ & $15 / 30(50 \%)$ \\
\hline
\end{tabular}

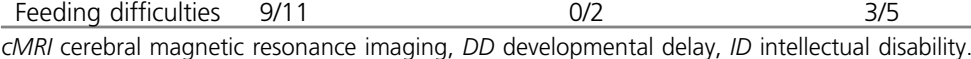

a Patient numbers are given only for those where the respective information was available, therefore the total count can be lower than the number of patients harboring the respective variant.

bIncluding relative microcephaly.

CYFIP2 is indicated to be intolerant to both missense $(z=$ 6.01) and loss-of-function (probability of LoF intolerance $[\mathrm{pLI}]=1)$ variants in gnomAD. However, no such LoF sequence variants or small structural aberrations have been reported in patients so far. It remains unclear if the putative LoF variants detected in our three individuals cause or contribute to the observed phenotype.

\section{Structural modeling of the missense variants predicts effects on WRC or CYFIP2 stability}

We mapped each of the previously and currently reported CYFIP2 missense variants onto the available crystal structure of human WRC and classified the predicted effects on CYFIP2 stability and CYFIP2 affinity to its interaction partners (Supplementary Table S2). Our estimates of changes in binding affinity within the WRC predicted a significant reduction of the binding affinity for 15 of 20 variants that could be modeled. As we have reported previously, Arg87 and Asp724 are located at the interface to WASF1 in the three-dimensional protein fold (Fig. 2a, b $)^{5}$ and form direct intermolecular interactions to a6and C-helices of WASF1. CYFIP2 variants affecting these recurrently mutated positions disturb these interactions and therefore favor the release of the activity-bearing VCA domain of WASF1. Substitutions of Arg87 are additionally predicted to cause destabilization of the CYFIP2 structure due to a loss of intramolecular interactions. Met456 and Tyr690 are both located in the interface with NCKAP1 (Fig. 2a, c). The predominant effect of the p.(Tyr690Cys) is on the interaction with NCKAP1, whereas p.(Met456Val) is predicted to mainly affect the stability of CYFIP2 itself (Supplementary Table S2). The interaction of Glu468 with BRICK1 is likely weakened by the p.(Glu468Asp) variant (Fig. 2a, d). Met311 and Phe888 are buried in the CYFIP2 structure (Fig. 2a, e, f). The variants affecting these residues result in disturbed interactions in the protein core and thus probably impact CYFIP2 stability. The p.(His1206Tyr) exchange is not expected to significantly affect protein stability or WRC interactions. However, His1206 is close to the mapped RAC1 D binding site of CYFIP, ${ }^{14}$ and we therefore speculate that this variant influences WRC activation by affecting RAC1-CYFIP2 interaction. The effects of p.(Thr490Met) could not be modeled due to the incomplete structural information in the respective region of the crystal structure.

\section{Missense variants impair dorsal ruffle formation in patient fibroblasts}

As our structural modeling predicted the CYFIP2 variants to affect WASF activity, we investigated the effect on actin cytoskeleton reorganization upon growth factor stimulation by staining filamentous actin with phalloidin in fibroblast cultures of all six consenting affected individuals. We did not observe apparent differences in lamellipodia formation. 
a

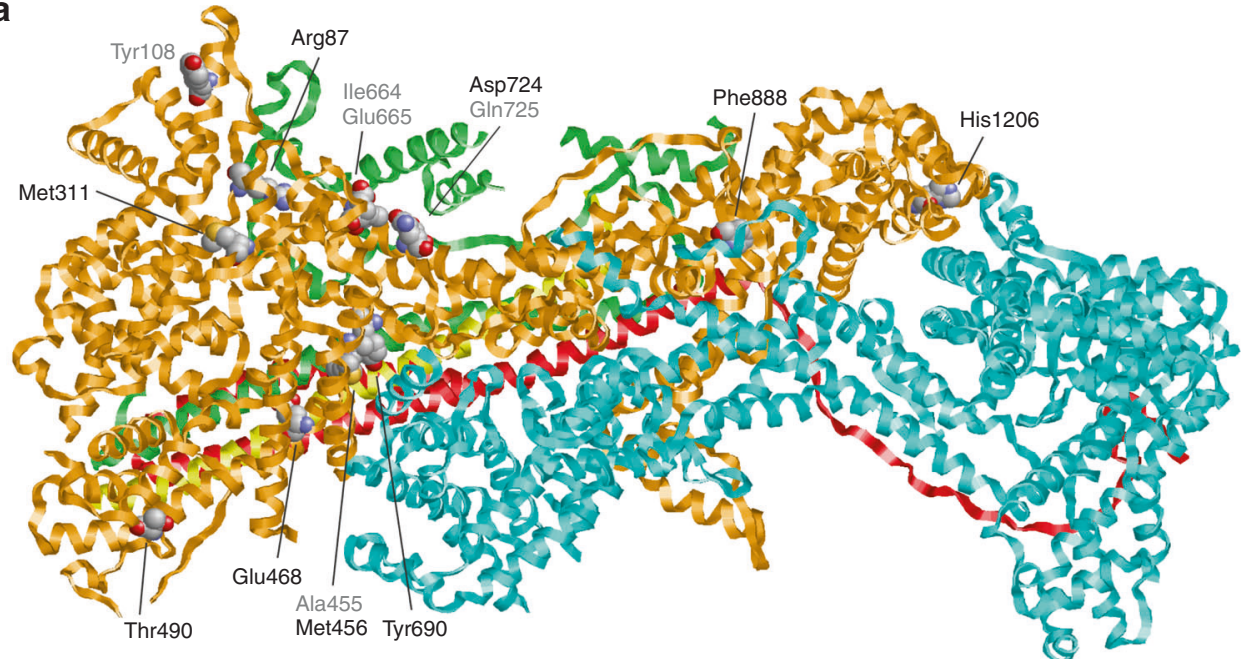

b

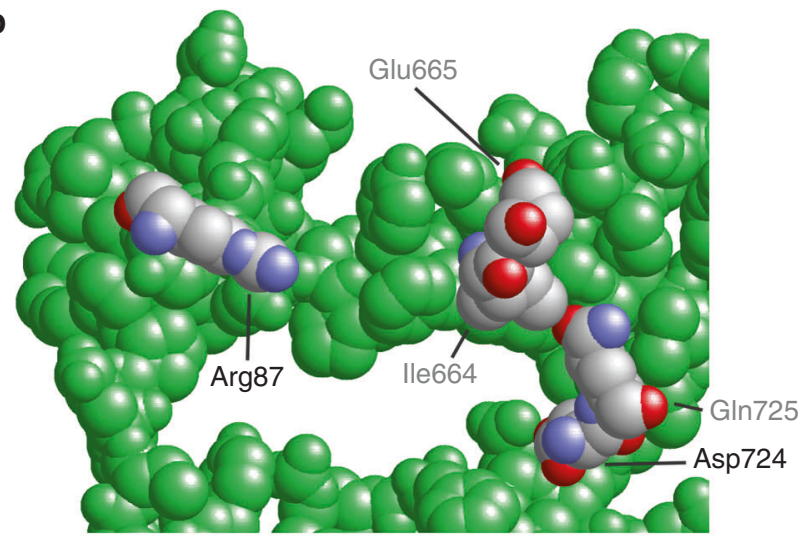

d

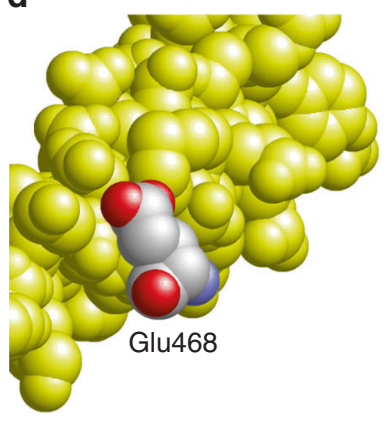

e

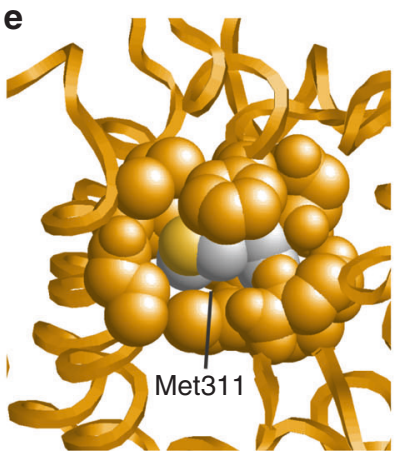

C

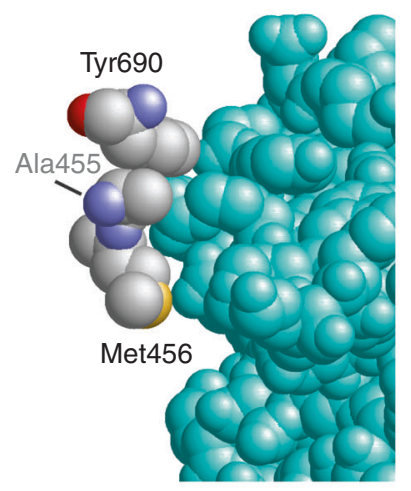

f

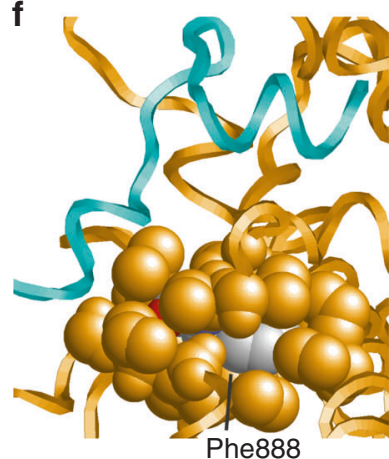

Fig. 2 Structural location of CYFIP2 missense variants. (a) Structure of the WAVE-regulatory complex. The individual protein components are shown in ribbon presentation and colored as follows: CYFIP2 (orange), NCKAP1 (cyan), WASF1 (green), BRICK1 (yellow), and ABI2 (red). Residues for which missense variants were detected are shown in space-filled presentation and are colored according to the atom type. Black and gray labels denote residues identified in the present and in previous studies, respectively. The same coloring scheme is also used for (b-f), which show enlargements of structurally relevant regions. (b) Arg87, lle664, Glu665, Asp724, and Gln725 (all colored according to their atom types) are located in the interface with WASF1 (green space-filled presentation). (c) Ala455, Met456, and Tyr690 are all located in the interface with NCKAP1 (cyan space-filled presentation). (d) Glu468 is located in the interface with BRICK1 (yellow space-filled presentation). (e) Met311 is buried in the CYFIP2 structure. All atoms closer than $5 \AA$ to Met311 are shown in orange space-filled presentation. (f) Phe888 is buried in the CYFIP2 structure (orange) close to the interaction site of NCKAP1 (cyan ribbon). All atoms closer than $5 \AA$ to Phe888 are shown in space-filled presentation.

However, a significantly reduced number of circular dorsal ruffles (CDRs) was observed in all patient fibroblast lines compared with fibroblasts of three controls (Fig. 3). CDRs are ring-shaped, F-actin-rich structures that are transiently formed in response to growth factor stimulation. ${ }^{15}$ As CDRs are implicated in preparation for cell movement, we further investigated a possible effect of CYFIP2 variants on fibroblast migration using a wound healing assay. A slower migration 


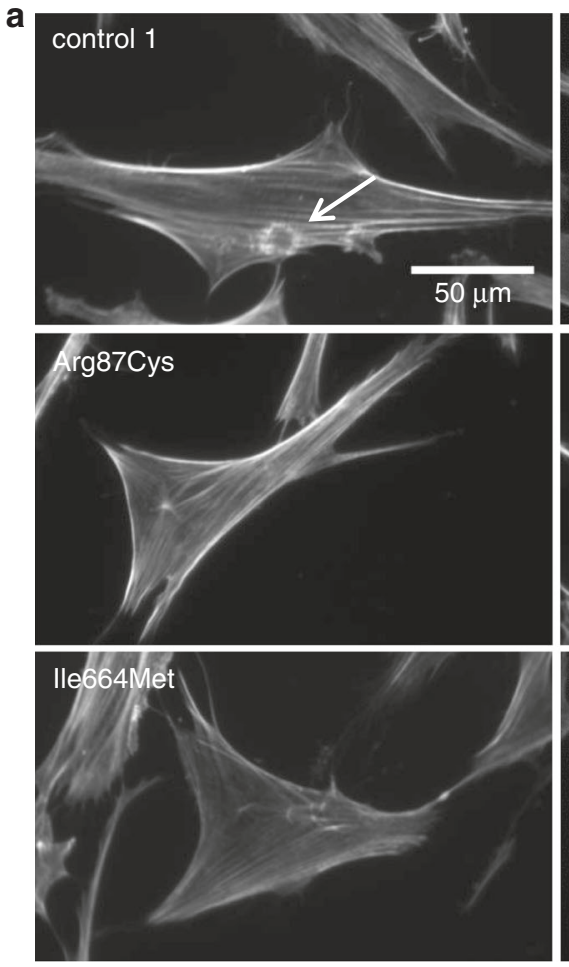

b

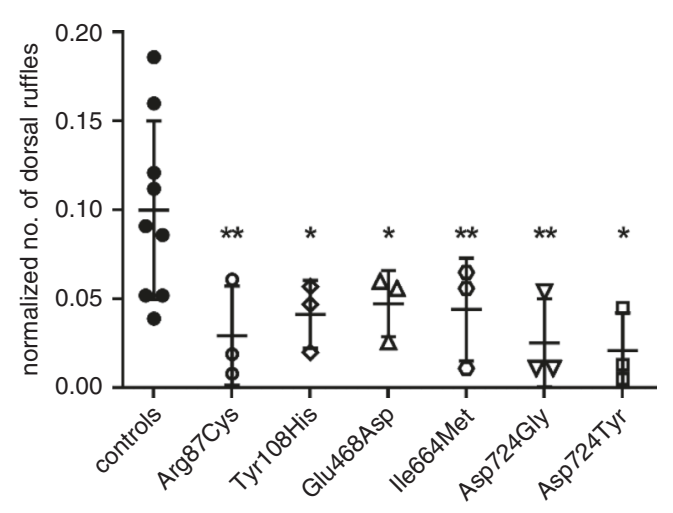

d

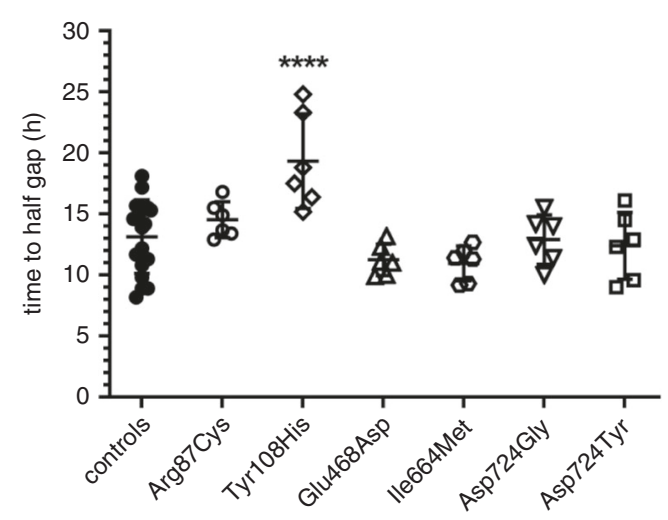

speed and consecutively an increased time to half gap closure was observed only for the variant p.(Tyr108His), while there was no difference in migration for the other patient fibroblasts (Fig. 3).

C
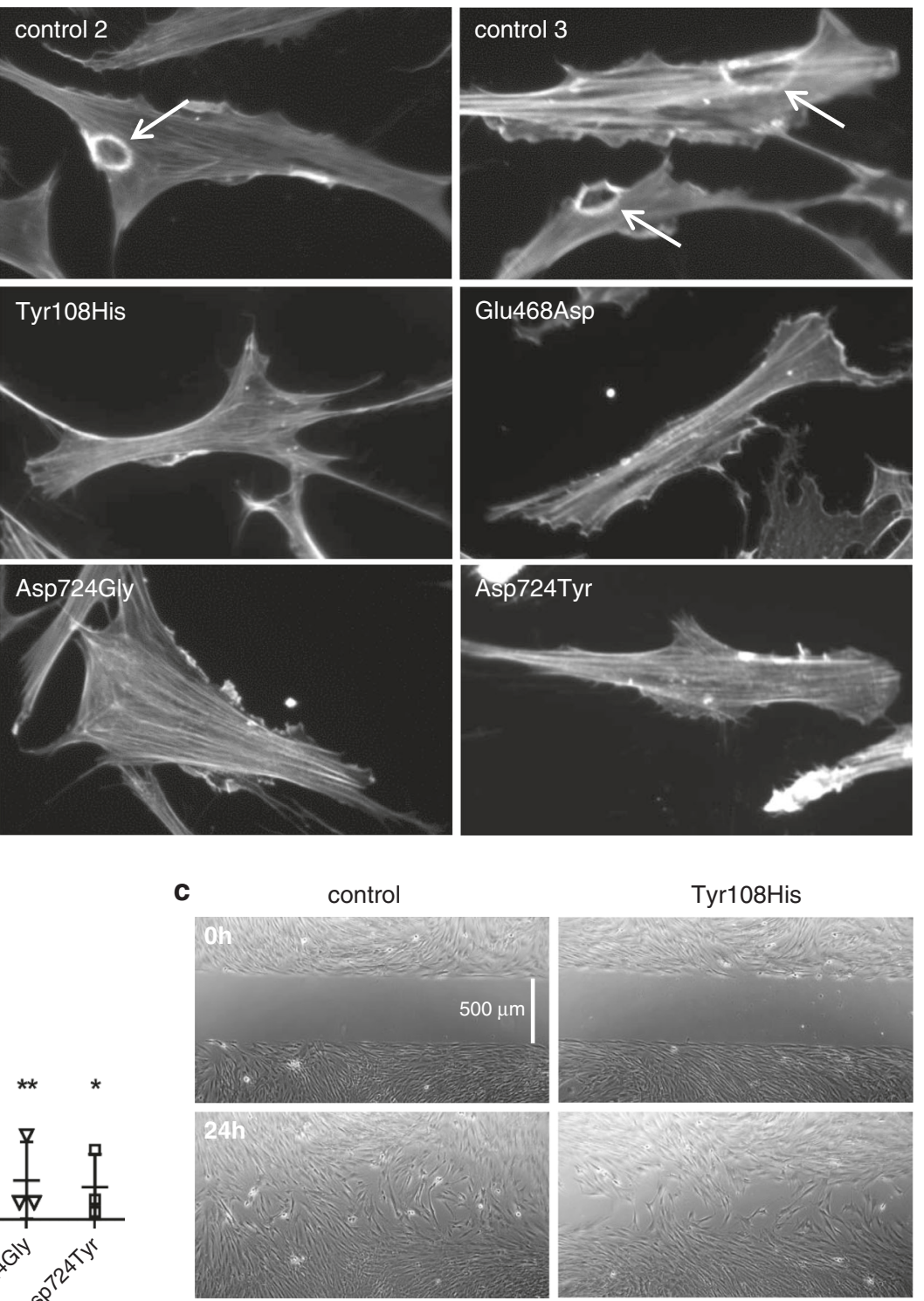

Tyr108His
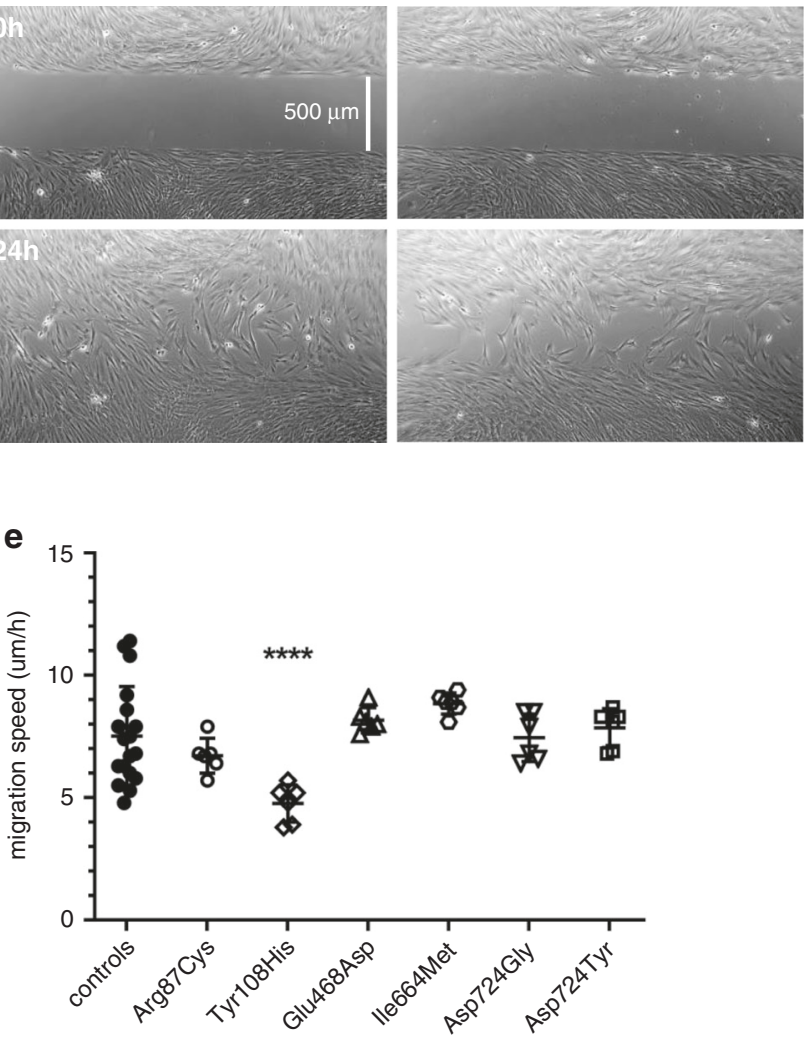

DISCUSSION

Our data on 16 new individuals harboring likely diseaseassociated CYFIP2 missense variants and three individuals harboring LoF variants of unknown significance considerably 
Fig. 3 Phenotype in primary fibroblasts of affected individuals heterozygous for pathogenic CYFIP2 missense variants and unaffected controls. (a) Representative images of PDGF-stimulated primary fibroblasts with phalloidin staining of F-actin. Fewer dorsal ruffles (indicated with white arrows) were observed in patient cells compared with unaffected control cells. (b) Quantification of dorsal ruffle formation in patient and control fibroblasts. The number of counted dorsal ruffles was normalized to cell number. Three independent experiments are shown, and at least 200 cells were analyzed in each. (c) Representative images of wound healing assays with fibroblasts carrying the p.(Tyr108His) variant and a control line at time points 0 hours and 24 hours. (d,e) Wound healing assay analysis of (d) time to half gap and (e) migration speed of patient and control fibroblasts. Three independent experiments with two technical replicates each were analyzed. Only fibroblasts harboring the $\mathrm{p}$.(Tyr108His) variant showed a significant impairment of migration compared with controls. Mean and standard deviation are displayed, and statistical significance was evaluated using one-way analysis of variance (ANOVA) with $p$ values indicated as follows: ${ }^{*} p<0.05,{ }^{*} p<0.01, p^{* * * *}<0.0001$.

broaden and delineate the genetic and phenotypic spectrum of the novel CYFIP2-related neurodevelopmental disorder. The new individuals with CYFIP2 missense variants presented with similar features as reported previously, including ID/DD in all cases, and seizures, muscular hypotonia, and microcephaly frequently observed (Table 2 , Supplementary Table S1) ${ }^{4-7}$ By reporting on data of five further individuals harboring a variant at the mutational hotspot p.Arg87, including two novel substitutions, we confirm that p.Arg87 individuals show a consistently very severe phenotype of developmental and epileptic encephalopathy. In addition, we establish the aspartate at protein position 724 as a novel recurrently affected residue in CYFIP2 by describing four individuals harboring three different substitutions. However, the total of five Asp724 individuals (including patient 11 in Zweier et al. ${ }^{5}$ ) present with a more variable phenotype ranging from profound ID with intractable epilepsy to moderate ID without epilepsy. In addition to the recurrently affected sites, we contribute seven individuals with private variants affecting novel positions in CYFIP2. Of note, two of these individuals (I13 and I14 harboring variants p.(Tyr690Cys) and p. (Met456Val), respectively) showed normal motor development milestones with a delay of language development only, considerably widening the phenotypic spectrum of the CYFIP2-related neurodevelopmental disorder.

It has been previously shown that CYFIP2 missense variants spatially cluster in the tertiary structure and are predicted to weaken the interaction with WASF1 or NCKAP1 leading to enhanced WASF1 activation. ${ }^{5}$ We add six novel variants located at the WASF1 or NCKAP1 interface and describe the CYFIP2-BRICK1 interaction interface as a disease-associated site affected by variant (p.(Glu468Asp)) (Fig. 2). Such destabilization of the WRC due to disturbed protein-protein interactions has already been experimentally supported by demonstrating weaker interaction of CYFIP2 p.Arg87 variants with the VCA of WASF1, the active domain that is usually sequestered by CYFIP2 binding. ${ }^{4}$ In total, 15 of the 21 variants observed so far are predicted to impair the interaction with WRC members (Supplementary Table S2). Another effect predicted by our structural modeling is a destabilization of the CYFIP2 protein itself, which may result in impaired interaction with the components of the WRC complex. A change of CYFIP2 stability is predicted for 13 of the 21 variants reported. Interestingly, lower protein levels of the CYFIP2 p.(Ala455Pro) and p.Arg87 variants compared with wild type and other tested CYFIP2 mutants have already been observed in an overexpression model. ${ }^{16}$ However, in a single LCL cell line established from a patient harboring the $p$. (Arg87Leu) variant no changes in CYFIP2 levels were detected. ${ }^{4}$ Nevertheless, changes of expression levels of CYFIP proteins in neurons were reported to alter spine morphology and the excitatory/inhibitory balance and neuronal excitability, ${ }^{17,18}$ which are considered to be one of the key mechanisms underlying epilepsy and other neurodevelopmental disorders. Taken together, structural modeling suggests that the mutated CYFIP2 proteins lead to impaired WRC stability and hereby to increased WASF1 activity. The only variant that is predicted to have no relevant impact on binding affinity or CYFIP2 stability is p.(His1206Tyr). However, this variant is located near the RAC1 D binding site of CYFIP. ${ }^{14}$ Mutants of the D binding site have been shown to result in a loss of RAC1 binding and impaired morphology and function of actin-based cell protrusions. ${ }^{14,19}$ Hence, we assume that the p.(His1206Tyr) variant may affect actin polymerization through a distinct pathomechanism. Notably, the phenotype of the individual (I16) with moderate ID and no seizures was not apparently different from the other individuals.

To substantiate the effect of CYFIP2 variants on WASF1 activity in vitro we investigated actin cytoskeleton reorganization in six patient fibroblast lines, which carried variants at the WASF1 or BRICK1 interaction interface. In all fibroblast lines an impaired formation of CDRs was observed (Fig. 3), a process already shown to require WASF1 activity. ${ }^{15}$ Actin dynamics and CDRs are important for cell movement. ${ }^{20,21}$ However, we did not observe significant differences in cell migration of patient fibroblasts in a wound healing assay, with the exception of the cells harboring the previously reported ${ }^{5}$ variant p.(Tyr108His) (Fig. 3). This inconspicuous result for five of six tested variants could be due to limited sensitivity in the patient fibroblast model, which has lower CYFIP2 expression than the nervous system and still expresses a fully functional protein from the wild-type allele. However, fibroblasts carrying the p.(Tyr108His) variant did exhibit slower migration. The respective individual, published as patient 12 in Zweier et al., showed profound ID and epilepsy and no clearly distinct features. Interestingly, Tyr108 is annotated as a phosphorylation site with strong evidence in PhosphoSitePlus, ${ }^{22}$ which is lost in the Tyr108His substitution. Signals from many different pathways including Rac GTPase, phospholipids, and protein kinases converge at the different subunits of the WRC to regulate its membrane 
localization and activation at the right time. Accordingly, phosphorylation and dephosphorylation of CYFIP2 and the WASF proteins have been shown to influence the release of the VCA domain, actin polymerization, and dendritic spine formation in neurons. Such phosphorylation sites include Thr138 and Tyr151 of WASF1 that are located in the vicinity of CYFIP2 Tyr108 at the WASF1 meander region. ${ }^{2,23-25}$ Hence, it can be speculated that lack of phosphorylation at the mutated Tyr108 impairs signal integration and hereby WRCdependent cell motility, in addition to the disturbed dorsal ruffle formation observed.

CYFIP2 and its effector WASF1 (or their orthologs) are enriched in the brain ${ }^{26,27}$ and have been implicated in several aspects of neuronal morphogenesis, including morphology of synapses and neurites or axon pathfinding in a multitude of models. ${ }^{1,3,23,28-32}$ Variants in both CYFIP2 and WASF1 lead to a similar but unspecific neurodevelopmental phenotype with ID, seizures, and muscular hypotonia, as well as microcephaly, visual impairments and other features in some patients. ${ }^{5,33} R A C 1$, $A C T B$, and $A C T G 1$ variants have also been shown to result in similar overlapping phenotypes. ${ }^{34-36}$ Although the exact pathomechanisms are yet to be elucidated, the current evidence from fibroblast studies demonstrates an impairment of WRCdependent actin regulation, suggesting that a disturbed actin regulation signaling cascade explains the neurological phenotype of the patients in disorders linked with this pathway.

In addition to the reported CYFIP2 missense variants for which we claim pathogenicity, we also identified three putative loss-of-function variants. There is currently no clear evidence derived from databases of copy-number or sequence variants supporting pathogenicity or benignity of LoF variants in CYFIP2. The three individuals reported in this study harboring such variants also pose some challenges for interpretation of their clinical relevance. The segregation cannot be clarified in two cases, and in the third case a de novo inheritance is established but other family members were reported to have developmental difficulties indicating a possible other, shared cause for the phenotype. Additionally, no fibroblasts of individuals harboring LoF variants were available for in vitro assessment. A potential compensation of CYFIP2 loss of function by the highly homologous CYFIP1 seems unlikely as multiple lines of evidence suggest that they cannot compensate for each other; for example, their expression was found to show cell-type specific differences with Cyfip2 being mainly expressed in neurons, while Cyfip1 expression levels were higher in non-neuronal cells in the mouse brain. ${ }^{27}$ Moreover, homozygous knockout in mice for either gene is lethal, and Cyfip1 and Cyfip2 are expressed at different developmental time points, ${ }^{29,37-39}$ and distinct functions have been described in animal models. ${ }^{29,31,40}$

However, as all three individuals with putative LoF variants have a milder phenotype than the individuals with de novo missense CYFIP2 variants we conclude that the complete loss of one allele product does not lead to the severe neurodevelopmental phenotype found in cases with pathogenic missense variants.
In conclusion, our data of 19 new individuals harboring CYFIP2 variants considerably broadens the genetic and phenotypic spectrum of the novel CYFIP2-related neurodevelopmental disorder typically caused by de novo missense variants, while we propose that loss of one allele product leads to a milder phenotype or may not be disease causing. Our findings in patient fibroblasts indicate that pathogenic heterozygous CYFIP2 missense variants disturb the WRC and consecutively the Arp2/3-induced actin polymerization that is required for the concise temporal and spatial regulation of CDR formation. In line with previous findings, ${ }^{4,5}$ we conclude that the underlying mechanism is a disinhibition of WASF1 by aberrant CYFIP2, resulting in dysregulation of the WAVEregulatory pathway.

Further work is needed to understand the functional consequences of CYFIP2 variants on WASF activity and actin dynamics, and to elucidate their effect on the FMRP interaction and translation regulation not studied so far. Additionally, future observations of CYFIP2 loss-of-function variants or small deletions in humans will help to elucidate the question of CYFIP2 haploinsufficiency.

\section{SUPPLEMENTARY INFORMATION}

The online version of this article (https://doi.org/10.1038/s41436020-01011-x) contains supplementary material, which is available to authorized users.

\section{ACKNOWLEDGEMENTS}

The authors are grateful to the participating individuals and their families. We thank Isabelle Rouvet from the Center for Cell Biotechnology of the Lyon University Hospital for fibroblast cultures of patient 12 published in Zweier et al. ${ }^{5}$ Imaging and migration assay analysis was performed with support of the Center for Microscopy and Image Analysis, University of Zurich. A. R. was supported by the Swiss National Science Foundation (SNSF) grant 320030_179547. A.R. and H.S. were supported by the Clinical Research Priority Program of the University of Zurich (CRPP Praeclare). A.B. was supported by the Forschungskredit Candoc by the University of Zurich grant FK-18-025. The DDD Study (Cambridge South REC approval 10/H0305/83 and the Republic of Ireland REC GEN/284/12) presents independent research commissioned by the Health Innovation Challenge Fund (grant number HICF-1009-003), a parallel funding partnership between the Wellcome Trust and the Department of Health, and the Wellcome Trust Sanger Institute (grant number WT098051). The views expressed in this publication are those of the author(s) and not necessarily those of the Wellcome Trust or the Department of Health. K.Õ. and T.R. are supported by the Estonian Research Council grant PRG471.

\section{DISCLOSURE}

K.M. and A. Begtrup are employees of GeneDx, Inc. The other authors declare no conflicts of interest. 
Publisher's note Springer Nature remains neutral with regard to jurisdictional claims in published maps and institutional affiliations.

\section{REFERENCES}

1. Schenck A, Bardoni B, Langmann C, et al. CYFIP/Sra-1 controls neuronal connectivity in Drosophila and links the Rac1 GTPase pathway to the fragile $X$ protein. Neuron. 2003;38:887-898.

2. Chen Z, Borek D, Padrick SB, et al. Structure and control of the actin regulatory WAVE complex. Nature. 2010;468:533-538.

3. Schenck A, Qurashi A, Carrera P, et al. WAVE/SCAR, a multifunctional complex coordinating different aspects of neuronal connectivity. Dev Biol. 2004;274:260-270.

4. Nakashima M, Kato $M$, Aoto $K$, et al. De novo hotspot variants in CYFIP2 cause early-onset epileptic encephalopathy. Ann Neurol. 2018;83:794-806.

5. Zweier M, Begemann A, McWalter K, et al. Spatially clustering de novo variants in CYFIP2, encoding the cytoplasmic FMRP interacting protein 2, cause intellectual disability and seizures. Eur J Hum Genet. 2019;27:747-759.

6. Peng J, Wang $Y$, He F, et al. Novel West syndrome candidate genes in a Chinese cohort. CNS Neurosci Ther. 2018;24:1196-1206.

7. Zhong M, Liao S, Li T, et al. Early diagnosis improving the outcome of an infant with epileptic encephalopathy with cytoplasmic FMRP interacting protein 2 mutation: case report and literature review. Medicine (Baltimore). 2019;98:e17749.

8. Sobreira N, Schiettecatte F, Valle D, Hamosh A. GeneMatcher: a matching tool for connecting investigators with an interest in the same gene. Hum Mutat. 2015;36:928-930.

9. Sayle RA, Milner-White EJ. RASMOL: biomolecular graphics for all. Trends Biochem Sci. 1995;20:374.

10. Xiong $P$, Zhang $C$, Zheng W, Zhang $Y$. BindProfX: assessing mutationinduced binding affinity change by protein interface profiles with pseudocounts. J Mol Biol. 2017:429:426-434.

11. Delgado J, Radusky LG, Cianferoni D, Serrano L. FoldX 5.0: working with RNA, small molecules and a new graphical interface. Bioinformatics. 2019;35:4168-4169.

12. Berg $S$, Kutra $D$, Kroeger $T$, et al. ilastik: interactive machine learning for (bio)image analysis. Nat Methods. 2019;16:1226-1232.

13. McQuin C, Goodman A, Chernyshev V, et al. CellProfiler 3.0: nextgeneration image processing for biology. PLoS Biol. 2018;16:e2005970.

14. Chen B, Chou HT, Brautigam CA, et al. Rac1 GTPase activates the WAVE regulatory complex through two distinct binding sites. eLife. 2017;6: e29795.

15. Suetsugu S, Yamazaki D, Kurisu S, Takenawa T. Differential roles of WAVE1 and WAVE2 in dorsal and peripheral ruffle formation for fibroblast cell migration. Dev Cell. 2003;5:595-609.

16. Lee $Y$, Zhang Y, Ryu JR, et al. Reduced CYFIP2 stability by Arg87 variants Causing Human Neurological Disorders. Ann Neurol. 2019;86:803-805.

17. Pathania M, Davenport EC, Muir J, et al. The autism and schizophrenia associated gene CYFIP1 is critical for the maintenance of dendritic complexity and the stabilization of mature spines. Transl Psychiatry. 2014;4:e374.

18. Davenport EC, Szulc BR, Drew J, et al. Autism and schizophreniaassociated CYFIP1 regulates the balance of synaptic excitation and inhibition. Cell Rep. 2019;26:2037-2051

19. Schaks M, Singh SP, Kage F, et al. Distinct interaction sites of Rac GTPase with WAVE regulatory complex have non-redundant functions in vivo. Curr Biol. 2018;28:3674-3684.

20. Hoon JL, Wong WK, Koh CG. Functions and regulation of circular dorsal ruffles. Mol Cell Biol. 2012;32:4246-4257.

21. Innocenti M. New insights into the formation and the function of lamellipodia and ruffles in mesenchymal cell migration. Cell Adh Migr. 2018;12:401-416.

22. Hornbeck PV, Zhang B, Murray B, et al. PhosphoSitePlus, 2014: mutations, PTMs and recalibrations. Nucleic Acids Res. 2015;43:D512-D520.

23. Kim Y, Sung JY, Ceglia I, et al. Phosphorylation of WAVE1 regulates actin polymerization and dendritic spine morphology. Nature. 2006;442: 814-817.
24. Mendoza MC. Phosphoregulation of the WAVE regulatory complex and signal integration. Semin Cell Dev Biol. 2013;24:272-279.

25. Lee $Y$, Kim D, Ryu JR, et al. Phosphorylation of CYFIP2, a component of the WAVE-regulatory complex, regulates dendritic spine density and neurite outgrowth in cultured hippocampal neurons potentially by affecting the complex assembly. Neuroreport. 2017;28:749-754.

26. Sossey-Alaoui K, Head K, Nowak N, Cowell JK. Genomic organization and expression profile of the human and mouse WAVE gene family. Mamm Genome. 2003;14:314-322.

27. Zhang Y, Kang HR, Han K. Differential cell-type-expression of CYFIP1 and CYFIP2 in the adult mouse hippocampus. Anim Cells Syst (Seoul). 2019;23:380-383.

28. Zallen JA, Cohen $Y$, Hudson AM, et al. SCAR is a primary regulator of Arp2/3-dependent morphological events in Drosophila. J Cell Biol. 2002;156:689-701.

29. Han K, Chen H, Gennarino VA, et al. Fragile X-like behaviors and abnormal cortical dendritic spines in cytoplasmic FMR1-interacting protein 2-mutant mice. Hum Mol Genet. 2015;24:1813-1823.

30. Pittman AJ, Gaynes JA, Chien CB. nev (cyfip2) is required for retinal lamination and axon guidance in the zebrafish retinotectal system. Dev Biol. 2010;344:784-794.

31. Cioni JM, Wong HH, Bressan D, et al. Axon-axon interactions regulate topographic optic tract sorting via CYFIP2-dependent WAVE complex function. Neuron. 2018;97:1078-1093.

32. Shakir MA, Jiang K, Struckhoff EC, et al. The Arp2/3 activators WAVE and WASP have distinct genetic interactions with Rac GTPases in Caenorhabditis elegans axon guidance. Genetics. 2008;179:1957-1971.

33. Ito $Y$, Carss KJ, Duarte ST, et al. De novo truncating mutations in WASF1 cause intellectual disability with seizures. Am J Hum Genet. 2018;103:144-153.

34. Reijnders MRF, Ansor NM, Kousi M, et al. RAC1 missense mutations in developmental disorders with diverse phenotypes. Am J Hum Genet. 2017;101:466-477.

35. Cuvertino S, Stuart HM, Chandler KE, et al. ACTB loss-of-function mutations result in a pleiotropic developmental disorder. Am J Hum Genet. 2017;101:1021-1033.

36. Riviere JB, van Bon BW, Hoischen A, et al. De novo mutations in the actin genes $A C T B$ and ACTG1 cause Baraitser-Winter syndrome. Nat Genet. 2012;44:440-444.

37. Zhang $Y$, Kang $H$, Lee $Y$, et al. Smaller body size, early postnatal lethality, and cortical extracellular matrix-related gene expression changes of Cyfip2-null embryonic mice. Front Mol Neurosci. 2018;11:482.

38. Kumar V, Kim K, Joseph $\mathrm{C}$, et al. C57BL/6N mutation in cytoplasmic FMRP interacting protein 2 regulates cocaine response. Science. 2013;342:1508-1512.

39. Chung L, Wang $X$, Zhu L, et al. Parental origin impairment of synaptic functions and behaviors in cytoplasmic FMRP interacting protein 1 (Cyfip1) deficient mice. Brain Res. 2015;1629:340-350.

40. Bozdagi O, Sakurai T, Dorr N, et al. Haploinsufficiency of Cyfip1 produces fragile X-like phenotypes in mice. PLoS One. 2012;7:e42422.

Open Access This article is licensed under a Creative Commons Attribution-NonCommercial-ShareAlike 4.0 International License, which permits any non-commercial use, sharing, adaptation, distribution and reproduction in any medium or format, as long as you give appropriate credit to the original author(s) and the source, provide a link to the Creative Commons license, and indicate if changes were made. If you remix, transform, or build upon this article or a part thereof, you must distribute your contributions under the same license as the original. The images or other third party material in this article are included in the article's Creative Commons license, unless indicated otherwise in a credit line to the material. If material is not included in the article's Creative Commons license and your intended use is not permitted by statutory regulation or exceeds the permitted use, you will need to obtain permission directly from the copyright holder. To view a copy of this license, visit http://creativecommons.org/licenses/by-nc-sa/4.0/.

(C) The Author(s) 2020 
Anaïs Begemann, $\mathrm{MD}^{1}$, Heinrich Sticht, $\mathrm{PhD}^{2}$, Amber Begtrup, PhD, FACMG ${ }^{3}$, Antonio Vitobello, $\mathrm{PhD}^{4,5}$, Laurence Faivre, $\mathrm{MD}, \mathrm{PhD}^{4,6}$, Siddharth Banka, MD, PhD ${ }^{7,8}$, Bader Alhaddad, MD ${ }^{9}$, Reza Asadollahi, MD, PhD ${ }^{1}$, Jessica Becker, PhD ${ }^{10}$, Tatjana Bierhals, MD ${ }^{11}$, Kathleen E. Brown, MS, CGC ${ }^{12}$, Ange-Line Bruel, PhD ${ }^{4,5}$, Theresa Brunet, MD ${ }^{9}$, Maryline Carneiro, $\mathrm{MD}^{13}$, Kirsten Cremer, $\mathrm{MD}^{10}$, Robert Day, $\mathrm{PhD}^{14}$, Anne-Sophie Denommé-Pichon, $\mathrm{MD}^{4,5}$, Dave A. Dyment, MD ${ }^{15,16}$, Hartmut Engels, PhD ${ }^{10}$, Rachel Fisher, MS, CGC ${ }^{17}$, Elaine S. Goh, MD ${ }^{18}$, M. J. Hajianpour, MD, $\mathrm{PhD}^{19}$, Lucia Ribeiro Machado Haertel, MD ${ }^{20}$, Nadine Hauer, MSc ${ }^{21}$, Maja Hempel, MD ${ }^{11}$, Theresia Herget, $\mathrm{MD}^{11}$, Jessika Johannsen, $\mathrm{MD}^{22}$, Cornelia Kraus, $\mathrm{PhD}^{21}$, Gwenaël Le Guyader, MD, PhD ${ }^{23}$, Gaetan Lesca, MD ${ }^{24,25}$, Frédéric Tran Mau-Them, MD, PhD ${ }^{4,5}$, John Henry McDermott, MD ${ }^{7,8}$, Kirsty McWalter, MS, $C_{G C}^{3}$, Pierre Meyer, MD ${ }^{26}$, Katrin Õunap, MD, PhD ${ }^{27,28}$, Bernt Popp, MD ${ }^{21,29}$, Tiia Reimand, MD, PhD ${ }^{27,28,30}$, Korbinian M. Riedhammer, MD ${ }^{9,31}$, Martina Russo, MSc ${ }^{1}$, Lynette G. Sadleir, MD ${ }^{32}$, Margarita Saenz, MD ${ }^{12}$, Manuel Schiff, MD ${ }^{33,34}$, Elisabeth Schuler, MD ${ }^{35}$, Steffen Syrbe, MD $^{35}$, Amelie Theresa Van der Ven, MD ${ }^{11}$, Alain Verloes, MD, PhD ${ }^{36,37}$, Marjolaine Willems, MD ${ }^{38}$, Christiane Zweier, MD, PhD ${ }^{21}$, Katharina Steindl, MD ${ }^{1}$, Markus Zweier, $\mathrm{PhD}^{1}$ and Anita Rauch, MD $\mathbb{1 D}^{1,39,40}$

\footnotetext{
${ }^{1}$ Institute of Medical Genetics, University of Zurich, Schlieren-Zurich, Switzerland; ${ }^{2}$ Institute of Biochemistry, Emil-Fischer Center, Friedrich-Alexander-Universität Erlangen-Nürnberg, Erlangen, Germany; ${ }^{3}$ GeneDx, Gaithersburg, MD, USA; ${ }^{4}$ INSERM UMR 1231 Equipe GAD, Université de Bourgogne, Dijon, France; ${ }^{5}$ Unité Fonctionnelle Innovation en Diagnostic génomique des maladies rares, FHU-TRANSLAD, CHU Dijon Bourgogne, Dijon, France; ${ }^{6}$ Centre de Référence Maladies Rares «Anomalies du développement et syndromes malformatifs", centre de génétique, FHU-TRANSLAD, CHU Dijon Bourgogne, Dijon, France; ${ }^{7}$ Manchester Centre for Genomic Medicine, St Mary's Hospital, Manchester University NHS Foundation Trust, Health Innovation Manchester, Manchester, UK; ${ }^{8}$ Division of Evolution \& Genomic Sciences, School of Biological Sciences, Faculty of Biology, Medicine and Health, University of Manchester, Manchester, UK; ${ }^{9}$ Institute of Human Genetics, Klinikum rechts der Isar, School of Medicine, Technical University of Munich, Munich, Germany; ${ }^{10}$ Institute of Human Genetics, University of Bonn, School of Medicine \& University Hospital Bonn, Bonn, Germany; ${ }^{11}$ Institute of Human Genetics, Universitätsklinikum Hamburg-Eppendorf, Hamburg, Germany; ${ }^{12}$ University of Colorado Anschutz Medical Campus, Children's Hospital Colorado, Aurora, CO, USA; ${ }^{13}$ Department of Neuropediatrics, Lyon University Hospital, Lyon, France; ${ }^{14}$ Cancer Research Laboratory, Department of Biochemistry, University of Otago, Dunedin, New Zealand; ${ }^{15}$ Department of Pediatrics, University of Ottawa, Ottawa, ON, Canada; ${ }^{16}$ Children's Hospital of Eastern Ontario Research Institute, University of Ottawa, Ottawa, ON, Canada; ${ }^{17}$ Division of Pediatric Genetics, Metabolism, and Genomic Medicine, Department of Pediatrics, University of Michigan, Arbor, MI, USA; ${ }^{18}$ Laboratory Medicine and Genetics and Institute for Better Health, Trillium Health Partners, Mississauga, ON, Canada; ${ }^{9}$ Department of Pediatrics, Medical Genetics, Quillen College of Medicine, East Tennessee State University, Johnson City, TN, USA; ${ }^{20} \mathrm{Hospital}$ Santa Catarina de Blumenau, Blumenau, Brazil; ${ }^{21}$ Institute of Human Genetics, Friedrich-Alexander-Universität Erlangen-Nürnberg (FAU), Erlangen, Germany; ${ }^{22}$ Department of Pediatrics, University medical center Hamburg-Eppendorf, Hamburg, Germany; ${ }^{23} \mathrm{CHU}$ de Poitiers, Poitiers, France; ${ }^{24}$ Department of Medical Genetics, Lyon University Hospital, Lyon, France; ${ }^{25}$ CNRS UMR 5292, INSERM U1028, Claude Bernard Lyon 1 University, Lyon, France; ${ }^{26}$ Department of Pediatric Neurology, CHU Montpellier, PhyMedExp, University of Montpellier, INSERM, CNRS, Montpellier, France; ${ }^{27}$ Department of Clinical Genetics, Institute of Clinical Medicine, University of Tartu, Tartu, Estonia; ${ }^{28}$ Department of Clinical Genetics, United Laboratories, Tartu University Hospital, Tartu, Estonia; ${ }^{29}$ Institute of Human Genetics, University of Leipzig Hospitals and Clinics, Leipzig, Germany; ${ }^{30}$ Department of Biomedicine, Institute of Biomedicine and Translational Medicine, University of Tartu, Tartu, Estonia; ${ }^{31}$ Department of Nephrology, Klinikum rechts der Isar, School of Medicine, Technical University of Munich, Munich, Germany; ${ }^{32}$ Department of Paediatrics and Child Health, University of Otago, Wellington, New Zealand; ${ }^{33}$ Reference center for Inborn Errors of Metabolism, Necker University Hospital, APHP, Université de Paris, Faculté de médecine Paris-Descartes, Paris, France; ${ }^{34}$ Institut Imagine, Inserm UMRS_1163, Paris, France; ${ }^{35}$ Department of Pediatrics, University Hospital Heidelberg, Heidelberg, Germany; ${ }^{36}$ UMR 1141 INSERM, Université Paris Diderot, Sorbonne Paris Cité, Paris, France; ${ }^{37}$ Genetics Department, AP-HP, Robert-Debré University Hospital, Paris, France; ${ }^{38}$ Département Génétique Médicale, CHRU de Montpellier, Montpellier, France; ${ }^{39}$ Zurich Center for Integrative Human Physiology, University of Zurich, Zurich, Switzerland; ${ }^{40}$ Neuroscience Center Zurich, University of Zurich and ETH Zurich, Zurich, Switzerland.
} 